INTERNATIONAL DESIGN CONFERENCE - DESIGN 2018

https://doi.org/10.21278/idc.2018.0400

\title{
PARAMETRIC MOVEMENT SYNTHESIS: TOWARDS VIRTUAL OPTIMISATION OF MAN- MACHINE INTERACTION IN ENGINEERING DESIGN
}

\author{
A. Wolf and S. Wartzack
}

\begin{abstract}
A parametric movement synthesis approach is introduced, which allows the adaptation of a synthesized movement to a specific use case by varying geometrical parameters. This parametric movement synthesis empowers the product developer to optimize products purely virtually in terms of ergonomics and (dis-)comfort by using musculoskeletal simulation. We developed such a parametric model for a lifting movement to analyse several scenarios. The results of the analyses show to what extent this procedure may support the product developer to improve the man-machine interaction of products.
\end{abstract}

Keywords: human centred design, musculoskeletal simulation, movement synthesis, ergonomics, design for $x(D f X)$

\section{Introduction}

Musculoskeletal simulations empower the product designer to optimise the physical man-machine interaction between the product he wants to design and the prospective users in such a way that the prospective users experience a comfortable product use.

This sentence summarizes the vision, which motivates the following contribution. Hereby, we want to focus on the physical/ physiological man-machine interaction between the entire human body and technical products - such as presented by Krüger and Wartzack (2014) - in order to improve product design regarding ergonomics, usability or (dis)comfort (Rasmussen, 2005). In current product developments, the physical man-machine interaction is usually not considered until the first prototype has been assembled. Of course, requirements regarding physiological aspects and ergonomics are taken into account, but these requirements are mainly derived from expertise, tables and normative standards like the EN 894-4 (European Committee for Standardization, 2010) or the VDI 2242 (Verein Deutscher Ingenieure, 1986). These recommendations are usually very general or specific and therefore support the product developer only to a certain extent. In the modern virtual environment of product development however, the developer wishes to reduce the need of prototypes, physical mock-ups and test iterations, by using digital models to gain information about the consequences of decisions, regarding product properties, in an early state (Vajna et al., 2009). Designing products in CAD systems, or analysing structure mechanics with FEM software is state of the art. In the same way, user centred requirements could be virtually evaluated by implementing a virtual interaction between the product and a human model in a virtual mock up (Miehling et al., 2013). This requires an advanced human model that allows a detailed simulation and analysis of arbitrary movements. Ergonomic digital human models (e.g. Siemens Jack or Human Builder) are already in use to evaluate human centred requirements 
(Mühlstedt and Spanner-Ulmer, 2009). These models are capable to perform specified analyses, like space requirements analyses, movement range analyses, visual analyses or rudimental biomechanical valuations for postures or predefined movements. Unfortunately, these models have certain limitations, especially regarding their ability to analyse arbitrary movements dynamically (Wagner et al., 2007). This restricts their general applicability, which is why the vast majority of product developments still have to rely on usability tests with physical mock-ups (Miehling et al., 2013). A much more advanced tool in this respect is musculoskeletal simulation, providing a full body biomechanical human model, which is dynamically analysable for arbitrary movements. Thus it is a mighty tool to gain insight to a vast amount of inner body forces (Lund et al., 2012) and has therefore a huge potential to support product development as a CAE tool. Although validated and professional musculoskeletal simulation tools are available since more than a decade, product developers do not currently use those to realise such a virtual interaction. To explain this fact, the standard workflow of musculoskeletal simulations has to be understood. Musculoskeletal simulation was primarily developed for clinical applications like gait analysis. With the approach of inverse dynamics (Zajac, 1993), muscular forces, as well as joint reaction forces are computable for arbitrary movements. These forces can give an indication of how comfortable or ergonomic a movement is, when interacting with a product. The kinematic data representing the movement, the external forces and moments, as well as the human multibody system and its boundary conditions (musculoskeletal model) are the input to the dynamic equations of motion (Glitsch and Baumann, 1997). Whilst the musculoskeletal model is delivered by the corresponding software toolssuch as the AnyBody Modeling System (Damsgaard et al., 2006) or OpenSim (Delp et al., 2007) - the movement as well as the external forces need to be measured in a specialised movement laboratory. External forces are primarily measured using force plates (Fluit et al., 2014). The measurement of movements is realisable via motion capturing techniques (Andersen et al., 2009). These preceding measurements require a physical mock-up, that makes the measurements expensive, time consuming and technically challenging (Farahani et al., 2016). Moreover, performing measurements conflicts with the idea of virtual product development. Especially the fact that any change in product design would lead to a new measurement of movement, to evaluate the new design towards human centred requirements, describes the impracticability of such measurements. This is the reason why the optimisation of physical man-machine interactions via musculoskeletal simulation is still a vision.

A solution to overcome this problem are predictive models (Farahani et al., 2011; Skals et al., 2016). Fundamentally, the idea is to predict the input data to avoid the need of measurements. Therefore, using inverse dynamics, a prediction of the movement as well as a prediction of the external forces is necessary. There are contact models, which enable external force predictions. Far more challenging is the prediction of dynamically consistent movements. The dynamic consistency of a movement implies that a movement is generally executable. Thus, dynamic consistency is necessary to guarantee a robust and valid inverse dynamic analysis and can consequently be understood as a measure of a successful synthesised (realistic/ plausible) movement. There are many approaches towards motion prediction that rather focus on generating plausible looking movements (e.g. for character animation in the film and gaming industry) than predicting dynamically consistent movements. One of those approaches is movement prediction via deep learning methods, which identify motion patterns using data mining on measured movement data (Holden et al., 2016). Approaches focusing on the synthesis of dynamically consistent movements usually use multibody simulation methods, since those contain the body dynamics. One such an approach (which is also applied in robotics) is movement prediction via optimal control of a forward dynamic analysis (Ackermann and van den Bogert, 2010). Those forward dynamic motion predictions are extremely demanding and usually only valid for a particular movement pattern (e.g. walking). Accordingly, the approach is currently not flexible enough to be universally applicable in product development. Another approach is inverse-inverse dynamics (Rasmussen et al., 2000). This approach wraps the inverse dynamic analysis in an optimisation problem, what makes the approach challenging in modelling and too computationally demanding for a useful application in product development (Wolf et al., 2017). Finally, there is the approach of Rousseau modelling, which specifies a movement via a definition of multiple time depended kinematical constraints. Rousseau models are the most suitable approach to match the needs of product development. This is due their relatively short 
calculation time, their simple and variable model creation as well as their ability to create dynamically consistent movements within the multibody domain of musculoskeletal simulation. (Wolf et al., 2017) The ability to predict input data is an important step towards integration of musculoskeletal simulations in product development. However, it is equally important to enable the developer to adapt the movement synthesis model via a simple and intuitive interface in order to synthesise a movement, matching the current product parameters. Rousseau models have a great advantage in this respect. Through a parametrisation of the necessary time-dependent kinematical constraints (usually defined for the end effectors), the movement becomes adaptable to a given design by simply adjusting few parameters. Under these preconditions, a parametric movement synthesis model would only have to be created once for a movement. If a similar movement shall be analysed, the product developer just needs to adapt the model for his specific product design. In this paper, we want to refer to this idea as parametric Rousseau models (PRM), which would allow a quick and easy use of musculoskeletal models. This way the analysis of a certain physical man-machine interaction becomes possible in an early state of product development and without the need of a physical mock up. In this contribution, however, we do not simply want to introduce the idea of PRMs, we also want to demonstrate how PRMs work and how they may support the product developer. To do so, a human lifting movement was synthesised, using Rousseau modelling. Through parametrisation, the Rousseau model was transformed into a PRM to analyse several fictional scenarios.

\section{Methods}

\subsection{Computational methods}

\subsubsection{Musculoskeletal Rousseau model}

A human lift movement shall be synthesised to analyse the lifting of an object to a higher place. The movement shall start shortly after the "object pick up" and last until the moment shortly before the "object put down". Therefore, the movement contains a "lifting phase" and a "put down phase". To synthesise this movement, a Rousseau model was developed using the AnyBody Modeling System and its musculoskeletal model from the AnyBody Managed Model Repository (AMMR) (version 1.6.6.).

Concrete values describing the joint angles define a suggested upright posture of the human model. Thus, an initial posture of the human model is determined forward kinematically. To drive the model, an introduction of additional external kinematical constraints is necessary (Figure 1). (Wolf et al., 2017)

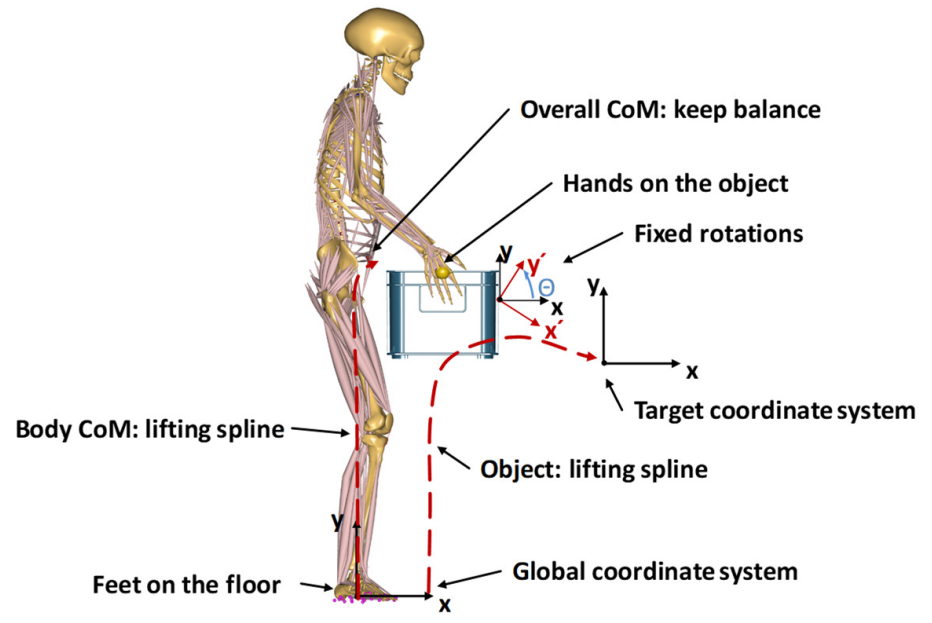

Figure 1. External constraints in order to synthesise the lifting movement

Obviously, the feet have to stay on the floor. Pseudo segments representing the object are constrained to be always in contact with the hands. Two B-splines describe the position of these pseudo segments over time. These splines are symmetrically to each other in relation to the $x-y-p l a n$ and describe the 
location from which the object lift starts and where it ends. The values in between are interpolated linearly. Fixed values describing the rotation of the pseudo segments ensure a correct orientation in space. In order to determine the execution of the lifting movement two further constraints are necessary. The first constraint fixes the overall centre of mass $(\mathrm{CoM})$ - including the pseudo segments - above the stand area between the feet. This constraint ensures a balanced movement. To determine whether the lifting movement performs from the legs or from the back, another spline constraint is required. This spline describes the location of the body's centre of mass - excluding the pseudo segments - over the time, by using a start and end height with a linear interpolation of the unknown values in between.

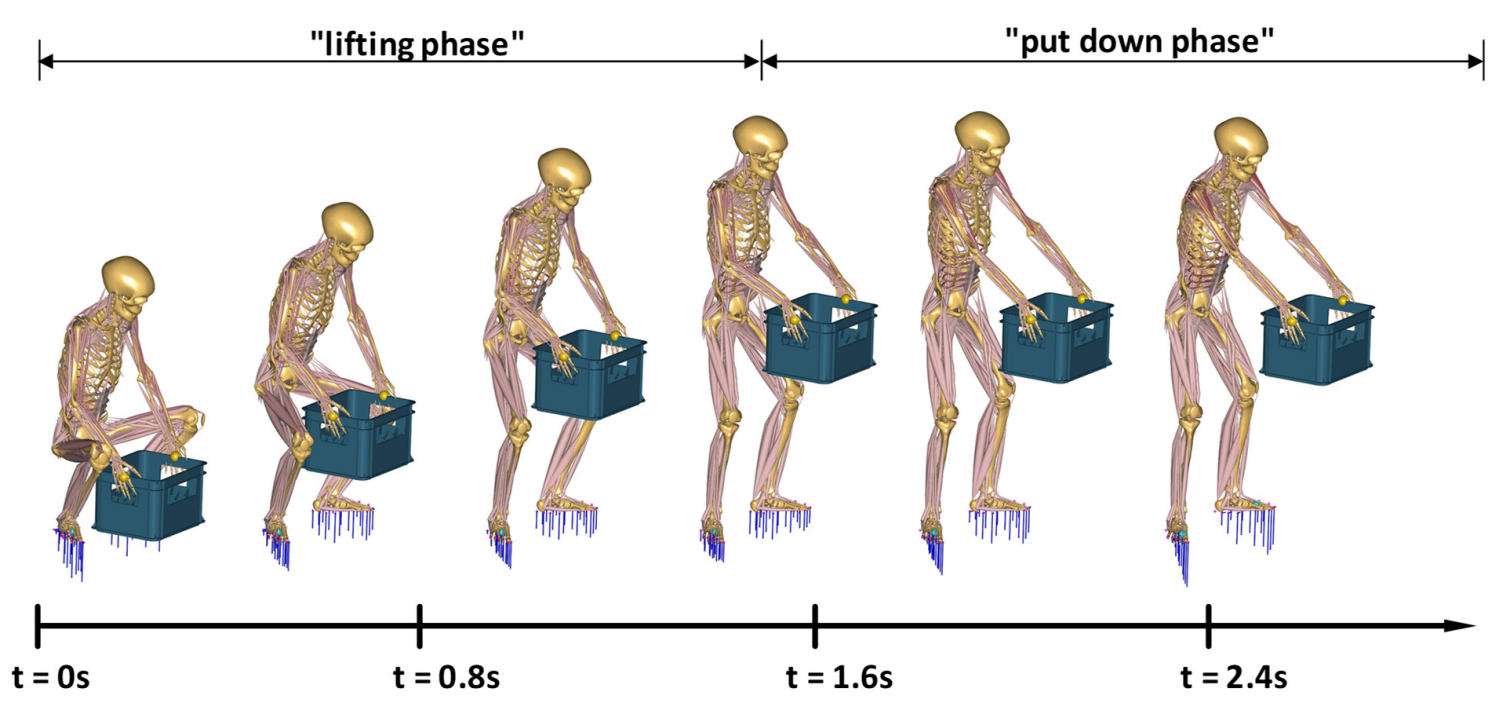

Figure 2. Synthesised lifting movement

The introduction of all these constraints leads to a kinematic over-determination of the human multibody system, since the specifications of the external constraints conflict with themselves and with the suggested joint angle values. In order to transform all these constraints into a movement, the method of over-determinate kinematics - which was introduced by Andersen et al. (2009) - is applicable. Originally, this method was developed for fitting motion-capturing data on the musculoskeletal model (which usually also is an over determinate case). The application of this method for Rousseau modelling is appropriate, since this fitting procedure also uses kinematical constraints. The method distinguishes between two types of constraints:

$$
\Gamma(q, t)=\left(\begin{array}{c}
\psi(q, t) \\
\phi(q, t)
\end{array}\right)
$$

The system coordinates $q$ to a certain time $t$ defined in the set of equations $\Psi(q, t)$ just have to be fulfilled as well as possible, whereas the equations defined in $\Phi(q, t)$ have to be solved exactly. For Rousseau models, it is useful to solve the constraints, which define the joints of the musculoskeletal model exactly. The constraints, which define the provisional joint angles as well as the external constraints just have to be fulfilled "as good as possible". Using this definition, an optimisation problem is able to find the best possible compromise between all the constraints:

$$
\text { min: } \quad G(\psi(q, t))
$$

$$
\text { subject to: } \phi(q, t)=0 \text {, }
$$

As an objective function, Anderson introduces a least square function with a time varying weight matrix:

$$
G(\psi(q, t))=\frac{1}{2} \psi(q, t)^{T} W(t) \psi(q, t),
$$


The weight matrix $W(t)$ is important for the application of Rousseau models, because it enables a prioritisation of various constraints. The suggested joint angles have a low priority, while the external constraints have a higher priority. This ensures a violation of the joint angles values, whilst the external constraints remain fulfilled as well as possible. This way, external constraints are able to drive the human model and therefore synthesise a movement. The movement's duration is defined with $2.8 \mathrm{~s}$, with a resolution of 10 steps per second. All these definitions synthesise the movement depictured in Figure 2.

\subsubsection{Inverse dynamics of the musculoskeletal system}

The Rousseau model provides the kinematical input for the inverse dynamic analysis in order to compute the muscle and joint reaction forces. The computation of the muscle forces however is not trivial. The number of muscles in the human body exceed the number of actuators that are strictly necessary to move the multibody system representing the human body. (Zajac, 1993; Heintz and Gutierrez-Farewik, 2007) Thus, the system is overdetermined, because infinite configurations of muscle forces could generate the prescribed movement. An optimisation problem may determine the "most realistic" configuration (Rasmussen et al., 2001).

$$
\begin{array}{ll}
\min : & G\left(f^{(M)}\right)=\sum_{i=1}^{n^{M}}\left(\frac{f_{i}^{(M)}}{N_{i}}\right)^{3} \\
\text { subject to: } & \mathbf{C f}=\mathbf{d} \\
& 0 \leq f_{i}^{(M)} \leq N_{i}, i \in\left\{1, \ldots, n^{(M)}\right\}
\end{array}
$$

The objective function is the polynomial sum over all muscle activities. The definition of muscle activity is the quotient of the actual force of a specific muscle $f_{\mathrm{i}}$ (design variables) and the maximum strength of this specific muscle $N_{\mathrm{i}}$. The optimisation problem is constrained by the dynamic equations of equilibrium, where $\boldsymbol{C}$ represents the coefficient matrix, $\boldsymbol{f}$ the unknown forces and $\boldsymbol{d}$ a vector of all known

loads. Equation (6) ensures that the muscles are unilateral actuators and that they have a restricted strengths $N_{\mathrm{i}}$. The implemented muscle model defines this muscle strength. The presented PRM uses the AnyMuscleModel.

\subsubsection{Prediction of external forces}

Beside the movement, the external forces are necessary to perform an inverse dynamic analysis. The external forces dynamically connect the musculoskeletal model with its environment. The most elementary contact is the one between the feet and the ground. To avoid measurements, a prediction of ground reaction forces is required. Fluit et al. (2014) did apply such a predictive contact model within the AnyBody Modeling System, which is also used for the present model. Imaginary residual forces and moments (acting on the pelvis) shall take over those force components the ground reaction forces cannot generate, due to dynamic inconsistencies. Since dynamic consistency is a measure of a successful synthesised movement, the height or the general occurrence of residual forces is also a measure of a successful movement synthesis. The contact model between the object and the hands consists of a hinge joint between the pseudo segments and the hands. The weight forces act on the hands according to the mass of the pseudo segments and are therefore more a declaration than a prediction.

\subsubsection{PRM of the human lifting movement}

The methods presented so far already allow an inverse dynamic analysis of one specific movement. The Rousseau model transforms into a PRM, when the product developer simply has to enter values for predefined parameters in order to define and consequently synthesise the desired movement. The parametrisation defines to which extent the movement shall be variable. Since product developers seldom have expertise regarding human movement patterns, the parameters should be purely geometrical in order to define the circumstances of the movement. The PRM itself shall cover the time dependencies as well as physiological aspects. In order to do so, the PRM bases upon the assumption that every lifting movement takes 2.8 seconds. This provides the required time restrictions. As a physiological assumption, the human model always fully straightens up when lifting 
the object, no matter how high the object shall be raised. The presented PRM shall enable an adjustment of the width and height of the handles on the object, as well as the objects' weight. In addition, it shall be possible to specify from which position, to which position the object needs to be lifted. Moreover, the developer shall be able to set whether the object has to be lifted over an edge or not. The developer may also chose, whether to lift from the back or from the legs. To obtain this variability of movement, certain parameters were introduced (Figure 3). These need to define the external constraints, driving the Rousseau model. The time dependent spline constraints are parameterised by a substitution of the control point values with the parameters. For example, the splines describing the position of the pseudo segments over time are expressed via the parameters object handle width, object handle height, lifting start anterior distance, lifting start height, lifting end anterior distance, lifting end height and edge height. The unknown control points in between are again interpolated linearly. Once the necessary external constraints are parameterised, the ordinary Rousseau model transforms into a PRM.

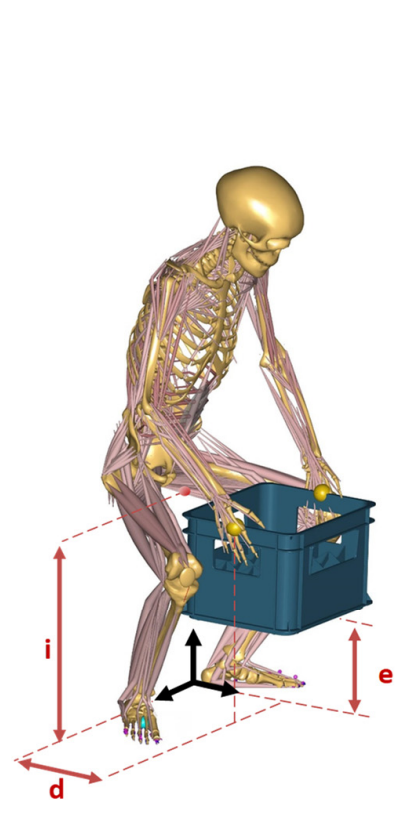

a)

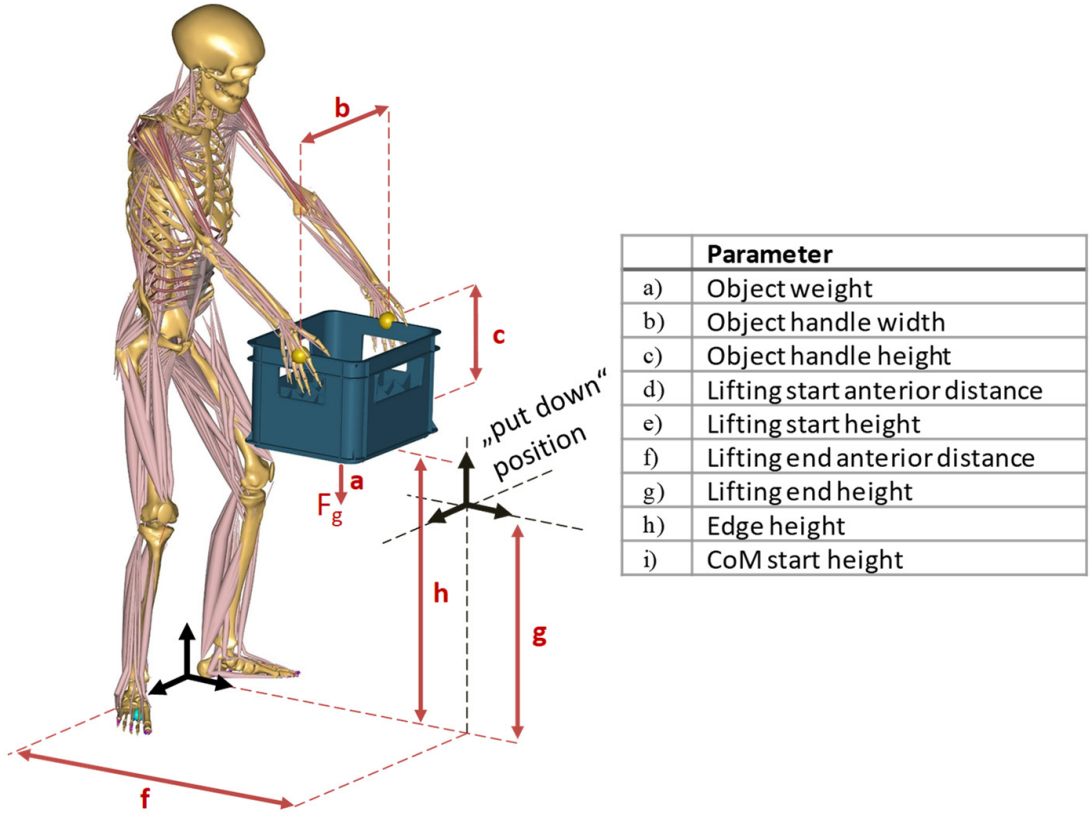

b)

Figure 3. Parametrisation of the lifting movement displayed for two time steps of the movement: a) First time step (start location of the object and the body-CoM); b) time step during lift over the edge (highest position of the object and final height of the body-CoM)

\subsubsection{Scaling}

In addition to the ability to adapt a movement to the desired case, the ability to scale the human model is important for the product developer. Since, every human being is an individual it is inadequate to use one model to describe all possible users. It is often sufficient to analyse the extreme values regarding the human anthropometrics (5th percentile/ 95 percentile). The PRM uses the Length-Mass-Fat Scaling (Rasmussen et al., 2005), which provides the possibility to scale the human model based on percentiles in order to adapt the strength, weight and body measurements.

\subsection{Experimental methods}

One intention of this contribution is to investigate whether the method of over-determinate kinematics (Andersen et al., 2009) is able to compute a dynamically consistent movement, for different constellations of parameter values for scaled models. If that is the case, the second fundamental question is whether and to which extent the gathered information's might support the product developer. To 
answer these questions the presented PRM of a lifting movement shall analyse several fictional scenarios. The scenarios describe use cases in which a product developer wants to improve his product design with the help of musculoskeletal models. This shall demonstrate the potential of PRMs in product development.

\subsubsection{Changing the environment - car boot study}

In the first scenario, the lifting of a filled beverage crate into a car boot shall be analysed. It might be interesting for the product developer to investigate how comfortable the boot of a certain car can be loaded. To prove whether the method of over-determinate kinematics is able to transform all the parameterised constraints of the PRM into a lifting movement, different constellations of parameter values are analysed as use cases (Table 1). To describe different kinds of users the human model is scaled to the 5th, 50th and 95th percentile per use case. The dimensions and the weight of object remain the same for all use cases (object weight $=10 \mathrm{~kg}$, object handle width $=0.4 \mathrm{~m}$, object handle height $0.25 \mathrm{~m})$.

Table 1. Overview of the varying parameters with the corresponding values

\begin{tabular}{|l|l|l|l|l|l|l|c|}
\hline \multicolumn{1}{|c|}{ Use cases } & $\begin{array}{c}\text { Lifting } \\
\text { start } \\
\text { anterior } \\
\text { distance }\end{array}$ & $\begin{array}{c}\text { Lifting } \\
\text { start } \\
\text { height }\end{array}$ & $\begin{array}{c}\text { Lifting } \\
\text { end } \\
\text { anterior } \\
\text { distance }\end{array}$ & $\begin{array}{c}\text { Lifting } \\
\text { end } \\
\text { height }\end{array}$ & $\begin{array}{c}\text { Edge } \\
\text { height }\end{array}$ & $\begin{array}{c}\text { CoM } \\
\text { start } \\
\text { height }\end{array}$ & Percentile \\
\hline 1. SUV-coupé, loading sill & $0.25 \mathrm{~m}$ & $0.00 \mathrm{~m}$ & $0.75 \mathrm{~m}$ & $0.73 \mathrm{~m}$ & $0.88 \mathrm{~m}$ & $0.48 \mathrm{~m}$ & 5 th $/ 50$ th $/ 95$ th \\
\hline 2. SUV-coupé, two-part tailgate & $0.25 \mathrm{~m}$ & $0.00 \mathrm{~m}$ & $0.55 \mathrm{~m}$ & $0.73 \mathrm{~m}$ & $0.73 \mathrm{~m}$ & $0.48 \mathrm{~m}$ & 5 th $/ 50$ th $/ 95$ th \\
\hline 3. middle class car & $0.25 \mathrm{~m}$ & $0.00 \mathrm{~m}$ & $0.55 \mathrm{~m}$ & $0.60 \mathrm{~m}$ & $0.60 \mathrm{~m}$ & $0.48 \mathrm{~m}$ & 5 th $/ 50$ th $/ 95$ th \\
\hline 4. lifting from a shopping cart & $0.30 \mathrm{~m}$ & $0.60 \mathrm{~m}$ & $0.35 \mathrm{~m}$ & $0.60 \mathrm{~m}$ & $0.80 \mathrm{~m}$ & $1.00 \mathrm{~m}$ & 5 th $/ 50$ th $/ 95$ th \\
\hline
\end{tabular}

In the first use case, a SUV-coupé car boot with a high loading sill is object of the analysis. The user has to lift the object over an edge (at the height of $0.88 \mathrm{~m}$ ) and therefore deep into the car boot. As expected, this process will not be very comfortable, especially for small users. To overcome this problem, the developers are considering a two-part tailgate for the same SUV coupé. Thus, the user would not have to lift over an edge and deep into the boot. This is the second use case that shall be analysed. For the sake of comparability, the boot of a middle class car, which is characterised by a low loading sill height, shall be analysed as use case 3 . As a fourth use case, the user shall lift the beverage crate from a shopping cart into the middle class car. The purpose of this use case is to evaluate the possible variability of the PRM. In reality, the user would do this by lifting the crate from the shopping cart, carrying it to the car, to put it down there. The PRM however is not able to model the part of the short walk (carrying) to the car. The use of the PRM is still acceptable, since the carrying part is negligible. This is because the strains during carrying are usually less significant than the strains during lifting and setting down. (Wilke et al., 2001)

In order to compare the different use cases, specific results of the inverse dynamic analysis are examined. The intradiscal pressure between the intervertebral discs L4 and L5 of the lumbar spine are particularly interesting for lifting movements (Bassani et al., 2017). To gain the intradiscal pressure from the compression force - which is the result of the inverse dynamic - the approach described by Nachemson (1960) is used, with a correction factor of 0,66 which is proposed from Dreischarf et al. (2013) and the cross sectional disc area from an MRI-scan presented by Wilke et al. (2001). As a second result, the distraction forces in the glenohumeral joint shall be an indicator for shoulder pain (Stuelcken et al., 2010). A further interesting result is the energy resulting from the metabolic costs, which are necessary to operate the given movement. The total metabolic costs are the sum of each muscle's metabolism. For the observations of this contribution, it is sufficient to calculate the muscle's metabolism by solely using the mechanical work of the muscle. Via numerical integration, the energy is obtainable from the total metabolic costs (Anderson and Pandy, 2001). 


\subsubsection{Changing the object - design of a road case}

In the first scenario, the movement had to adapt to a changing environment, while the lifted object remained the same. In the second scenario, the opposite situation is analysed, where the environment (from where, to where and how to lift) remains the same, while the parameters describing the object change. This may be of interest if the developer wants to design an object that is to lift frequently. One such object are road cases, which stage technicians have to work with. The developer may want to know which handles shall be chosen and where they shall be located, in order to make the lifting movement as comfortable as possible. For this purpose the parameters object handle width, as well as the object handle height of the road case are variable. For the sake of comparability, also the object weight is variable. (Table 2). In a small parameter study, each of the twelve possible combinations of discrete values are investigated to determine the best combination/ the best design. For comparability, the same results of the inverse dynamic analysis are used as for the first scenario.

Table 2. Overview of the varying parameters with the corresponding values

\begin{tabular}{|l|l|l|l|}
\hline \multicolumn{1}{|c|}{ Parameters } & \multicolumn{3}{c|}{ Variation of the Values } \\
\hline Object weight & $7.5 \mathrm{~kg}$ & $10 \mathrm{~kg}$ & \\
\hline Object handle width & $0.4 \mathrm{~m}$ & $0.45 \mathrm{~m}$ & $0.5 \mathrm{~m}$ \\
\hline Object handle height & $0.25 \mathrm{~m}$ & $0.3 \mathrm{~m}$ & \\
\hline
\end{tabular}

\section{Results}

\subsection{Changing the environment - car boot study}

The synthesis of a dynamically consistent movement (the residual forces and moments have insignificantly low values during the whole movement) was successful for all use cases. Therefore, it was possible to perform an inverse dynamic analysis for all the synthesised movements to gain the intended results (Figure 4). There was only one exception. The movement synthesis did not complete for the fifth percentile human model in use case 1. As shown in a) d) and g), the simulation terminates shortly before the end (blue curve). This is not because the over-determinate kinematics solver is not able to compute the solution. It is because the fifth percentile of the human model is simply too small to lift a beverage crate into the SUV-coupés car boot. As illustrated in a), b) and c), the glenohumeral distraction force has the highest values for use case 1 and the lowest values for use case 4 . In addition, tall humans have less strain in the shoulder than small ones when exercising the same movement. This behaviour was expectable. The intradiscal pressure displayed in d), e) and f), however shows a behaviour that was not expectable, since the pressure is higher for use case 2 and 3 than for use case 1 during the lifting phase. The values of use case 1 are only higher when the model has to lift the box deep into the boot, during the put down phase. This behaviour is explainable by looking at the assumptions that were necessary to create the PRM. Since both movements take the same time, and the human model fully straightens up in both movements, the box has a different position (height) in relation to the human model during the different movements. This position seems to be physiologically better in use case 1, regarding the intradiscal pressure. As a second effect, the crate has to accelerate more in use case 1 than in use case 2 or 3 . This leads to higher inertia forces, which relieve the strain in the back. The muscles however perform this acceleration, which explains why the movement in use case 1 requires more energy than in use case 2 or use case 3 . The figures g), h) and i) show the necessary energy, ascending over the time. They also unveil that tall persons have higher metabolic costs when performing the same movement, than small persons do. While the results of use case 1, 2 and 3 have similar curves, the results of use case 4 show a completely different, approximately constant curve. Due to the nearly constant and low strain, the lifting movement from a shopping cart is presumably the most comfortable one.

All this information may support the product developer to improve his product design. As the charts demonstrate, it is obvious that a two-part tailgate (use case 2) would be a benefit for the SUV coupé. When using the design considered in use case 1, the target group of small users would be excluded, as they would not be able to load the trunk with a full beverage crate. 


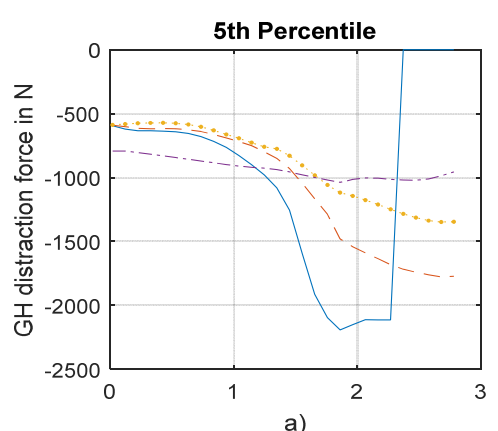

a)
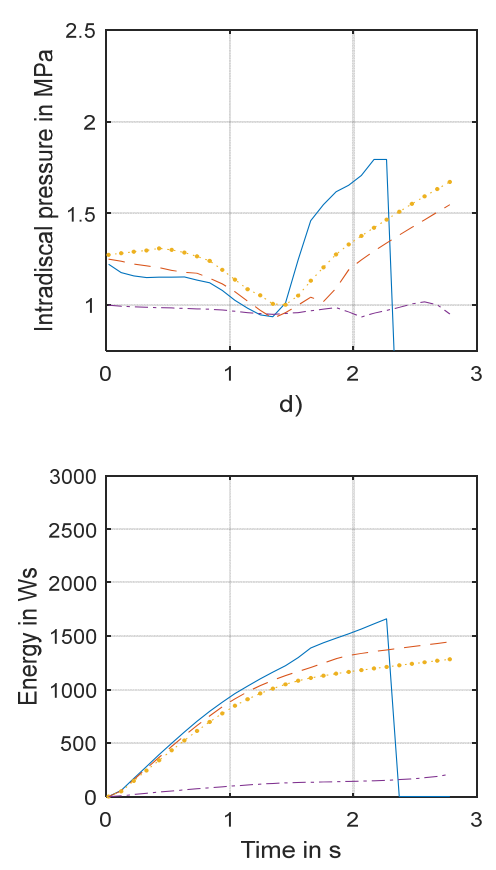

g)

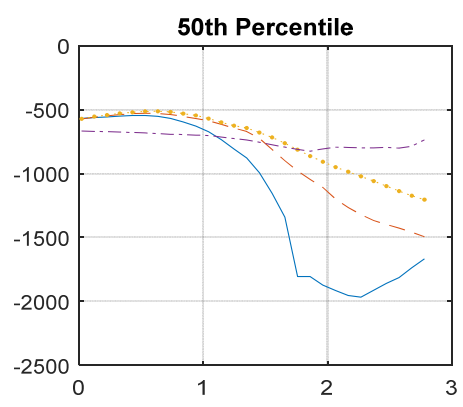

b)

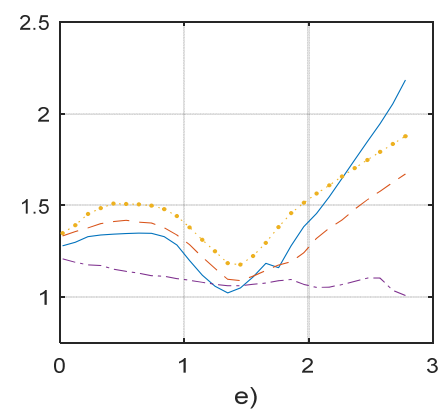

e)

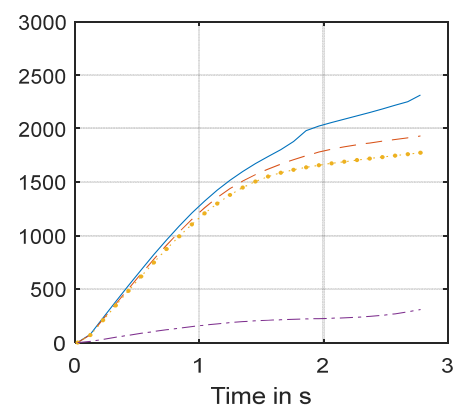

h)

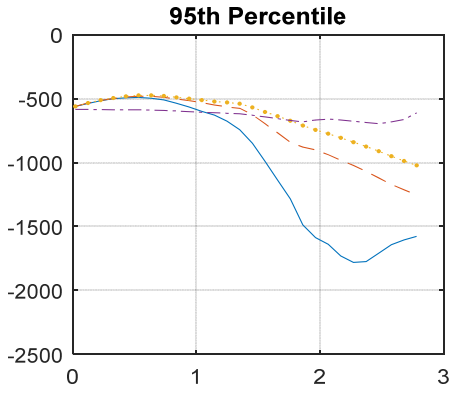

c)
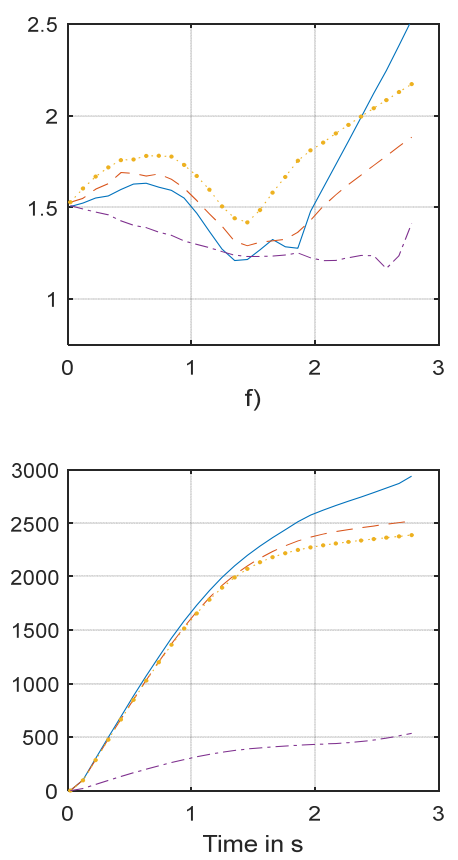

i)

use case $1--$ use case $2 \cdots \cdots$ use case $3-\cdots$ use case 4

Figure 4. Results of the inverse dynamics analysis for all use cases. Time histories of the glenohumeral distraction force, the intradiscal pressure and the energy for the different percentiles of the human model

\subsection{Changing the object - design of a road case}

The second scenario shall unveil how a changed geometry and weight of the objecot influence the movement and therefore the muscle and joint reaction forces. Again, it was possible to synthesise dynamically consistent movements and therefore analyse them inverse dynamically. As displayed in Figure 5, the parameter "object handle height" has major influence on the values' magnitudes, whilst the parameter "object handle width" has minor influence. This is an explainable behaviour, since higher located handles lead to a higher lifting height of the hands. The "object weight" parameter has more influence on the values' magnitudes than on the course of the curve. These information would hardly support the product developer, since an object handle height of $0.25 \mathrm{~m}$ leads to a lower intradiscal pressure, but in return to a higher glenohumeral distraction force (Figure $5 \mathrm{a}$ ),b),c),d)). The energy is slightly higher for a handle height of $0.3 \mathrm{~m}$, but the difference is negligible. This scenario shows that PRMs work robust, but it much more reveals the limitations of PRMs. The model executes the movement synthesis based on the kinematical inputs, but does not consider possible dynamic effects. In reality, a higher weight of the object would influence the way the object is lifted (course of the curve). In addition, the handle height would have a higher influence, since it affects the object's inertia forces acting on the hands. 

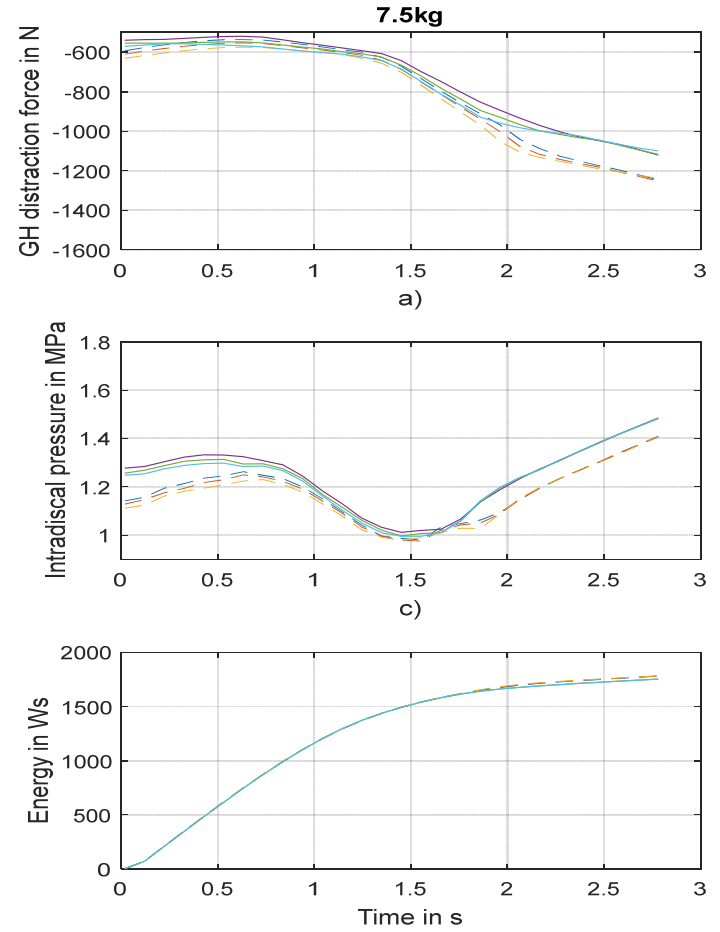

$\frac{e)}{--\mathrm{h}=0.3, \mathrm{w}=0.4-\mathrm{h}=0.3, \mathrm{w}=0.45-\mathrm{h}=0.3, \mathrm{w}=0.5}$

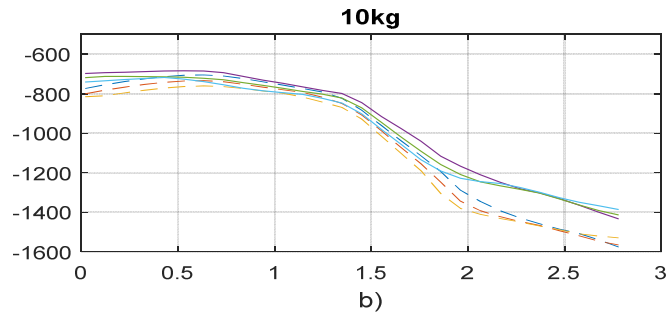

b)
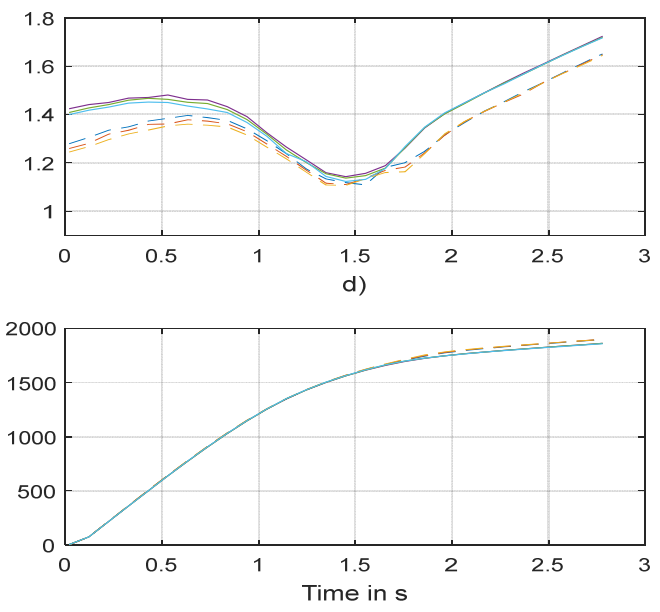

f)

Figure 5. Results of the inverse dynamics analysis for all combinations of scenario 2

\section{Discussion and conclusion}

The two scenarios unveil the potential of PRMs. Once a PRM is created, the product developer is able to adapt the model via a simple interface to synthesise a movement, matching his product parameters. In addition, PRMs are able to synthesise dynamically consistent movements, which can subsequently be analysed using inverse dynamics. The first scenario gives a glimpse of the possible variability of PRMs and to which extent the results may support the product developer in order to evaluate and improve product design. The second scenario reveals the limitations of PRMs. Since the creation of the movement is purely kinematical, it is roughly possible to cope with dynamic effects. Therefore, PRMs rather synthesise movements, than predict them. In other words, it is possible to model kinematical effects as well as prior known dynamic effects (e.g. the CoM-constraint who ensures dynamical balance), but the hope to find or model unknown dynamic effects cannot be fulfilled. The results however are plausible for the made assumptions, which indicates that the model works robust and reproducible. The magnitude of the computed intradiscal pressure matches with the results Wilke et al. (2001) have measured and Bassani et al. (2017) have simulated for a comparable use case. It is an impressive finding that the results' magnitudes are comparable with the real measurements by Wilke. Although the computed magnitudes are plausible and the synthesised movements are dynamically consistent, further scientific research need to prove the validity of the approach regarding the "physiological realism" of the movement itself and the inverse dynamically computed values.

In order to evaluate a product design using muscular forces, the muscle model AnyMuscleModel3E based on the Hill model (Hill, 1938) shall be applied instead of the AnyMuscleModel, since it delivers more realistic results. An enhanced realism of the muscle forces however, is not primarily important for this research, wherefore the use of the simpler muscle model is sufficient.

The developed PRM enables the revelation of inner body forces for various symmetric lifting movements, which allows a valuation of the different movements' (dis)comfort. The unveiled behaviour however was foreseeable to some extent. The challenge for future research will be to build more advanced, complicated and therefore less foreseeable models, to explore their benefits for product 
development. The PRM of the lifting movement could be parameterised in such a way, that a simple synthesis of an asymmetrical lifting movement or lifting with one hand becomes possible.

In addition to the synthesis of movements, PRMs can also be used to optimize dynamic inconsistent movements. For example, it would be possible to optimize or adapt movements that have been synthesized using deep learning methods in such a way that they become dynamically consistent and can therefore be analysed inverse dynamically.

The introductory sentence of this paper describes the vision, which motivates this contribution. Parametric Rousseau models could be the key to let this vision become reality. Because all inputs are synthesisable, an analysis of physical man-machine interaction becomes possible in a virtual mock-up, without the need for physical mock-ups or prototypes. PRMs do have certain limitations regarding dynamical effects but in exchange, the product developer obtains an easy, intuitive and computationally fast way to analyse his product design regarding (dis)comfort, ergonomics, efficiency and even safety.

\section{Acknowledgment}

This research and development work is funded by the "Bundesministerium für Wirtschaft und Technologie (BMWi)" within the program "Zentrales Innovationsprogramm Mittelstand (ZIM)". The project is supervised by the "Arbeitsgemeinschaft industrieller Forschungsvereinigungen (AiF)". At this point, the authors would like to appreciate the support and the funding. The authors are responsible for the content of this publication.

\section{References}

Ackermann, M. and van den Bogert, A.J. (2010), “Optimality principles for model-based prediction of human gait”, Journal of biomechanics, Vol. 43 No. 6, pp. 1055-1060. https://doi.org/10.1016/j.jbiomech.2009.12.012

Andersen, M.S., Damsgaard, M. and Rasmussen, J. (2009), "Kinematic analysis of over-determinate biomechanical systems", Computer methods in biomechanics and biomedical engineering, Vol. 12 No. 4, pp. 371-384. https://doi.org/10.1080/10255840802459412

Anderson, F.C. and Pandy, M.G. (2001), "Dynamic Optimization of Human Walking”, Journal of Biomechanical Engineering, Vol. 123 No. 5, p. 381. https://doi.org/10.1115/1.1392310

Bassani, T., Stucovitz, E., Qian, Z., Briguglio, M. and Galbusera, F. (2017), "Validation of the AnyBody full body musculoskeletal model in computing lumbar spine loads at L4L5 level”, Journal of biomechanics, Vol. 58, pp. 89-96. https://doi.org/10.1016/j.jbiomech.2017.04.025

Damsgaard, M., Rasmussen, J., Christensen, S.T., Surma, E. and de Zee, M. (2006), “Analysis of musculoskeletal systems in the AnyBody Modeling System", Simulation Modelling Practice and Theory, Vol. 14 No. 8, pp. 1100-1111. https://doi.org/10.1016/j.simpat.2006.09.001

Delp, S.L., Anderson, F.C., Arnold, A.S., Loan, P., Habib, A. et al. (2007), “OpenSim. Open-source software to create and analyze dynamic simulations of movement", IEEE transactions on bio-medical engineering, Vol. 54 No. 11, pp. 1940-1950. https://doi.org/10.1109/TBME.2007.901024

Dreischarf, M., Rohlmann, A., Zhu, R., Schmidt, H. and Zander, T. (2013), "Is it possible to estimate the compressive force in the lumbar spine from intradiscal pressure measurements? A finite element evaluation", Medical engineering \& physics, Vol.35 No. 9, pp. 1385-1390. https://doi.org/10.1016/j.medengphy.2013.03.007

European Committee for Standardization (2010), EN 894-4: Safety of machinery - Ergonomics requirements for the design of displays and control actuators - Part 4: Location and arrangement of displays and control actuators, Beuth Verlag.

Farahani, S.D., Andersen, M.S., de Zee, M. and Rasmussen, J. (2016), “Optimization-based dynamic prediction of kinematic and kinetic patterns for a human vertical jump from a squatting position", Multibody System Dynamics, Vol. 36 No. 1, pp. 37-65. https://doi.org/10.1007/s11044-015-9468-5

Farahani, S.D., Zee, M. de, Andersen, M.S., Lund, M.E. and Rasmussen, J. (2011), "Prediction of the movement patterns for human squat jumping using the inverse-inverse dynamics technique", XIII International Symposium on Computer Simulation in Biomechanics, Leuven, Belgium, 2011.

Fluit, R., Andersen, M.S., Kolk, S., Verdonschot, N. and Koopman, H.F.J.M. (2014), "Prediction of ground reaction forces and moments during various activities of daily living", Journal of biomechanics, Vol. 47 No. 10, pp. 2321-2329. https://doi.org/10.1016/j.jbiomech.2014.04.030

Glitsch, U. and Baumann, W. (1997), "The three-dimensional determination of internal loads in the lower extremity", Journal of biomechanics, Vol. 30 No. 11-12, pp. 1123-1131. https://oi.org/10.1016/S00219290(97)00089-4 
Heintz, S. and Gutierrez-Farewik, E.M. (2007), "Static optimization of muscle forces during gait in comparison to EMG-to-force processing approach", Gait \& posture, Vol. 26, pp. 279-288.

Hill, A.V. (1938), "The Heat of Shortening and the Dynamic Constants of Muscle", Proceedings of the Royal Society B: Biological Sciences, Vol. 126 No. 843, pp. 136-195. https://doi.org/10.1098/rspb.1938.0050

Holden, D., Saito, J. and Komura, T. (2016), "A deep learning framework for character motion synthesis and editing”, ACM Transactions on Graphics, Vol. 35 No. 4, pp. 1-11. https://doi.org/10.1145/2897824.2925975

Krüger D. and Wartzack S. (2014), "Towards CAD integrated simulation of use under ergonomic aspects", Proceedings of the DESIGN 20014 / 13th International Design Conference, Dubrovnik, Croatia, May 19-22, 2014, The Design Society, Glasgow, pp. 2095-2104.

Lund, M.E., de Zee, M., Andersen, M.S. and Rasmussen, J. (2012), “On validation of multibody musculoskeletal models", Proceedings of the Institution of Mechanical Engineers. Part H, Journal of engineering in medicine, Vol. 226 No. 2, pp. 82-94. https://doi.org/10.1177/0954411911431516

Miehling, J., Krüger, D. and Wartzack, S. (2013), "Simulation in Human-Centered Design - Past, Present and Tomorrow", In: Abramovici, M. and Stark, R. (Eds.), Smart Product Engineering, Lecture Notes in Production Engineering, Springer Berlin Heidelberg, Berlin, Heidelberg, pp. 643-652.

Mühlstedt, J. and Spanner-Ulmer, B. (2009), "Homo Sapiens Digitalis: über den Praxiseinsatz digitaler Menschmodelle", In: Lichtstein, A., Stößel, C. and Clemens, C. (Eds.), 8. Berliner Werkstatt MenschMaschine-Systeme: Der Mensch im Mittelpunkt technischer Systeme, VDI-Verlag, 2009.

Nachemson, A. (1960), "Lumbar Intradiscal Pressure. Experimental Studies on Post-Mortem Material", Acta Orthopaedica Scandinavica, Vol. 31 No. sup43, pp. 1-104. https://doi.org/10.3109/ort.1960.31.suppl-43.01

Rasmussen, J. (2005), "Musculoskeletal Simulation - (Dis)comfort Evaluation”, Sound \& vibration, Vol. 39 No. 5 , pp. 8-9.

Rasmussen, J., Damsgaard, M. and Christensen, S.T. (2000), "Inverse-inverse dynamics simulation of musculoskeletal systems", Proceedings of the 12th Conference of the European Society of Biomechanics, 2000, Royal Academy of Medicine in Ireland.

Rasmussen, J., Damsgaard, M. and Voigt, M. (2001), "Muscle recruitment by the $\min / \max$ criterion - a comparative numerical study", Journal of biomechanics, Vol.34 No.3, pp. 409-415. https://doi.org/10.1016/S0021-9290(00)00191-3

Rasmussen, J., de Zee, M., Damsgaard, M., Christensen, S.T., Marek, C. and Siebertz, K. (2005), “A general method for scaling musculoskeletal models", International Symposium on Computer Simulation in Biomechanics.

Skals, S., Jung, M.K., Damsgaard, M. and Andersen, M.S. (2016), "Prediction of ground reaction forces and moments during sports-related movements", Multibody System Dynamics, Vol. 39 No. 3, pp. 175-195. https://doi.org/10.1007/s11044-016-9537-4

Stuelcken, M.C., Ferdinands, R.E., Ginn, K.A. and Sinclair, P.J. (2010), "The Shoulder Distraction Force in Cricket Fast Bowling", Journal of Applied Biomechanics, Vol.26 No.3, pp. 373-377. https://doi.org/10.1123/jab.26.3.373

Vajna, S., Bley, H., Hehenberger, P., Weber, C. and Zeman, K. (2009), CAx für Ingenieure: Eine praxisbezogene Einführung, 2nd ed., Springer Berlin Heidelberg, Berlin, Heidelberg. https://doi.org/10.1007/978-3-54036039-1

Verein Deutscher Ingenieure (1986), VDI 2242: Konstruieren ergonomiegerechter Erzeugnisse, Grundlagen und Vorgehen, VDI, Düsseldorf.

Wagner, D.W., Reed, M.P. and Rasmussen, J. (2007), “Assessing the Importance of Motion Dynamics for Ergonomic Analysis of Manual Materials Handling Tasks using the AnyBody Modeling System", SAE Congress: Digital Human Modeling for Design and Engineering (DHM).

Wilke, H.-J., Neef, P., Hinz, B., Seidel, H. and Claes, L. (2001), "Intradiscal pressure together with anthropometric data - a data set for the validation of models", Clinical Biomechanics, Vol. 16, S111-S126. https://doi.org/10.1016/S0268-0033(00)00103-0

Wolf, A., Miehling, J. and Wartzack, S. (2017), "Vorgehensweisen zur Vorhersage menschlicher Bewegung durch muskuloskelettale Simulation”, In: Krause, D., Paetzold, K. and Wartzack, S. (Eds.), Design for X. Beiträge zum 28. DfX-Symposium, TuTech Verlag, Hamburg, pp. 13-24.

Zajac, F.E. (1993), "Muscle coordination of movement. A perspective", Journal of biomechanics, Vol. 26 No. 1 , pp. 109-124. https://doi.org/10.1016/0021-9290(93)90083-Q

Alexander Wolf, M.Sc.

Friedrich-Alexander-Universität Erlangen-Nürnberg, Chair of Engineering Design (KTmfk)

Martenstraße 9, 91058 Erlangen, Germany

Email: a.wolf@mfk.fau.de 